\title{
La educación después de la pandemia: propuesta de implementación de un modelo de Educación Básica a Distancia
}

\author{
Education after the pandemic: a proposal for the \\ implementation of a Distance Basic Education model
}

DOI: https://doi.org/10.32870/dse.v0i22.920

\author{
Zaira Navarrete Cazales* \\ Héctor Manzanilla Granados** \\ Lorena Ocaña-Pérez ${ }^{* * *}$
}

\begin{abstract}
Resumen
El cierre de los centros educativos en todo el mundo, provocado por la pandemia de COVID-19, ha obligado a replantearnos el papel de la escuela como un espacio físico, principalmente en lo que corresponde a la educación básica; en este sentido, el artículo presenta una propuesta de Educación Básica a Distancia a partir del análisis de las circunstancias que se están presentado en el terreno educativo derivado de dicha pandemia. Se da respuesta a las preguntas: ¿es posible estructurar un proyecto permanente de educación a distancia para la educación básica? ¿la modalidad a distancia permitiría una mayor inclusión y cobertura en la educación básica? y ¿es viable establecer la modalidad a distancia a partir de la educación básica primaria y secundaria? Se encontró que, en México, hasta la fecha no se ha implementado una modalidad de educación a distancia para el nivel básico de primaria y secundaria con el fin de dar mayor cobertura y apoyo a los alumnos, a pesar de ser una opción viable y probada en otros niveles educativos, por lo que en este texto se presenta una propuesta de Educación Básica a Distancia que pueda dar atención permanente a los alumnos que por distintas razones no asisten a la escuela de manera presencial, así como en casos de crisis como ocurre ahora por la pandemia de SAR-CoV-2.
\end{abstract}

Palabras claves: Educación a distancia - educación básica - cobertura - educación de calidad.

* Doctora en Ciencias. SNI I. Líneas de investigación: Formación profesional y construcción de identidades; Políticas y usos de TIC en educación; Historia y perspectiva de la pedagogía en México; Educación comparada e internacional; Agenda 2030 de la UNESCO. Profesora-investigadora de la Facultad de Filosofía y Letras, Pedagogía División SUAyED, Universidad Nacional Autónoma de México. México. znavarrete@filos. unam.mx

** Doctor en Ciencias. Líneas de investigación: Políticas y usos de TIC en educación; Educación Superior a Distancia. Profesor-investigador de la Escuela Superior de Cómputo del Instituto Politécnico Nacional (IPN). México. hmanzanilla@ipn.mx

*** Maestrante en Pedagogía. Líneas de investigación: Rezago educativo en la educación básica de México; Tecnologías de la Información y la Comunicación en educación. Profesora de la Facultad de Filosofía y Letras, Pedagogía División SUAyED, Universidad Nacional Autónoma de México. México. lorenaocana@filos.unam.mx 


\begin{abstract}
The closure of educational centers around the world caused by the COVID-19 pandemic has forced us to rethink the role of the school as a physical space, especially with regard to basic education. This article presents a proposal for Distance Basic Education based on an analysis of the circumstances faced by educators due to the pandemic. Is it possible to structure a permanent distance education project for basic education? Would the distance modality allow for greater inclusion and coverage in basic education? Is it feasible to establish a distance modality based on primary and secondary basic education? We found that, despite being a viable and proven option at other educational levels, a distance education modality that provides greater coverage and support to students for primary and secondary basic level has not yet been implemented in Mexico. This text presents a proposal for basic education at distance, which can provide ongoing attention to students who for various reasons do not attend school in person, as well as in crises such as the one provoked by the SARS-CoV-2 pandemic.
\end{abstract}

Keywords: Long distance education - basic education - coverage - quality education.

\title{
Introducción
}

En el año 2009 México pasó por una pandemia con el AH1N1, ${ }^{1}$ las medidas sanitarias solo incluyeron dos semanas de suspensión de clases en todas las escuelas a nivel nacional, hasta el 11 de mayo de 2009 (Salgado, Villavicencio, 2009). Las lecciones dejadas por la pandemia de AH1N1 no fueron asimiladas, la falta de infraestructura, presupuesto, protocolos y capacitación a los docentes para responder a una contingencia siguieron siendo cuentas pendientes en la agenda educativa de México (Ortega, 2020). La contingencia sanitaria originada por el Sars-CoV-2, causante del COVID-19, provocó un periodo de cuarentena mucho más extenso que el de 2009, en el que la escuela escolarizada ha acusado grandes deficiencias para cubrir las necesidades educativas de la población, a partir del cierre de las escuelas desde preescolar hasta el nivel superior.

La Organización Mundial de la Salud declaró el 11 de marzo de 2020 que el COVID-19 se considera una pandemia, por lo que pidió a los países afiliados que tomaran las medidas necesarias para controlarla (Adhanom, 2020). En este sentido, en México se emitió el Acuerdo número 12/03/20, por el que se determina la suspensión de clases en las escuelas públicas a partir del 23 de marzo y hasta el 17 de abril de 2020 (Moctezuma, 2020). Los términos de este acuerdo fueron modificados mediante un nuevo Acuerdo 06/03/20, en el que se extendió el periodo

1 La gripe por AH1N1 es una infección respiratoria aguda y muy contagiosa que proviende de los cerdos, causada por alguno de los varios virus gripales de tipo A de esa especie. La morbilidad suele ser alta, y la mortalidad baja (1\%-4\%). Se han notificado ocasionalmente brotes y casos esporádicos de infección humana por el virus de la gripe por AH1N1. En general los síntomas clínicos son similares a los de la gripe estacional, pero las manifestaciones clínicas son muy variables, desde una infección asintomática hasta una neumonía grave que mata al paciente (OMG, 2009: s/p).

Diálo Dos 
de suspensión escolar hasta el 30 de abril de 2020 (Moctezuma, 2020b). Posteriormente, dicho acuerdo también fue modificado, ahora por el Acuerdo 09/04/20, que extendió el periodo de suspensión hasta el 30 de mayo de 2020, especificando que las fechas quedarían sujetas a modificación por la autoridad competente (Moctezuma, 2020c). Pero ante la imposibilidad de un regreso seguro a las aulas, el 5 de junio de 2020 se publicó el Acuerdo 12/06/20, por el que el secretario de Educación Pública Esteban Moctezuma Barragán presentó las disposiciones para la evaluación del ciclo escolar 2019-2020, y declaró el fin del ciclo escolar a partir del 19 de junio de 2020 (Moctezuma, 2020d).

Aun no se cuenta con condiciones viables para un regreso seguro a la escuela, como lo declaró el secretario de Educación Pública en el Comunicado Conjunto $N^{\circ} 5$ del 9 de julio de 2020; el inicio del ciclo escolar 2020-2021 se realizará con base en el semáforo epidemiológico de cada entidad y solo cuando todas las actividades estén libres de restricciones sanitarias, con el fin de garantizar la seguridad, salud e higiene de todos los estudiantes y docentes del Sistema Educativo Nacional (Moctezuma, 2020e). Ante el panorama descrito, todavía no se tiene una idea clara de cómo será el regreso a las aulas, si se continuará con clases "a distancia" o si el regreso será con grupos reducidos; lo cierto es que se hace evidente la necesidad de establecer de manera permanente la modalidad de Educación a Distancia (ED) que pueda dar cobertura a la educación en los niveles de primaria y secundaria básica de México. ${ }^{2}$

Ante la incertidumbre del regreso a clases y la necesidad de hacer permanente la ED, se hace urgente dar respuesta a las preguntas: ¿es posible estructurar un proyecto permanente de educación a distancia para la educación básica? ¿la modalidad a distancia permitiría una mayor inclusión y cobertura en la educación básica? y ¿es viable establecer la modalidad a distancia a partir de la educación básica primaria y secundaria?

Con la intención de dar respuesta a estas preguntas es que el objetivo de este trabajo se centra en realizar un análisis del sitio web Aprende en Casa, propuesto por la Secretaría de Educación Pública (SEP), para fortalecerlo con el modelo instruccional de Gagné y Briggs. Todo ello con la finalidad de presentar una propuesta viable de Educación Básica a Distancia (EBD), que pudiera representar no solamente una herramienta de apoyo a la educación tradicional en situaciones de emergencia, sino que se configure en una modalidad permanente que permita llegar a sectores de la población que permanecen fuera de las escuelas por limitaciones físicas, culturales o económicas (Galeana, 2016).

2 En México la Educación Básica alude al proceso sistemático de la educación que comprende la instrucción preescolar ( 3 años de escolaridad; en promedio asisten niños de entre 3 a 5-6 años de edad), en la cual se imparten algunos conocimientos y se estimula la formación de hábitos; la instrucción primaria ( 6 años de escolaridad, en promedio asisten niños de entre 6 a 11-12 años de edad), en la cual se inician el conocimiento científico y las disciplinas sociales; y, por último, la instrucción secundaria ( 3 años de escolaridad, en promedio asisten jóvenes de entre 12 a 14-15 años de edad), en la que se amplían y reafirman los conocimientos científicos por medio de la observación, la investigación y la práctica (DGPP, 2008). 
El presente artículo es resultado de una investigación de corte cualitativo documental con sustento teórico-analítico, que analiza datos generados por autores y organismos nacionales, y la revisión de documentos emitidos por las autoridades educativas en México, informes y artículos relacionados con el tema de la educación básica durante la pandemia por COVID-19, así como del modelo instruccional propuesto para dar forma a un programa de Educación Básica a Distancia.

Este artículo se divide en tres apartados, en el primero se hace un análisis de la página web Aprende en Casa, propuesta por el gobierno federal para dar continuidad a la educación por motivo del cierre de escuelas como medida de aislamiento social debido a la pandemia de COVID-19 (Se analiza solo esta opción porque el tema de este trabajo es la implementación de la educación a distancia para los niveles de educación primaria y secundaria), así como las dificultades enfrentadas por los docentes, alumnos y padres de familia ante la educación mediada por las Tecnologías de Información y Comunicación (TIC), en relación con la falta de recursos, capacitación y acceso. En el segundo apartado se analizan las implicaciones de una propuesta de Educación Básica a Distancia: el aumento en la cobertura, aumento de la calidad educativa, la diversidad de esquemas pedagógicos que surgen de la modalidad a distancia, la oportunidad de fortalecer la investigación educativa, establecer que la educación a distancia debe considerarse un recurso valioso y no una finalidad en sí misma, y la evolución de los roles que asumen los actores educativos. En el tercer apartado se realiza una propuesta de implementación de EBD a través del modelo instruccional de Gagné y Briggs, ya que lo consideramos el más apropiado para dar inicio a un programa de este tipo, puesto que da la oportunidad de verificar y corregir cada etapa de un proyecto educativo a distancia. Finalmente, se vierten las conclusiones surgidas del presente trabajo.

\section{La educación en cuarentena: de las aulas a las pantallas}

Al inicio del aislamiento social, declarado por el gobierno federal a partir del 23 de marzo de 2020, se estableció la necesidad de continuar con la educación; se dedujo que la mejor opción para que los alumnos continuaran con sus estudios era la educación a distancia. Para llevar a cabo esta tarea, la SEP promovió la creación del sitio web Aprende en Casa ${ }^{3}$ (Moctezuma, Bucio, 2020: s/p), desestimando una realidad latente en la mayoría de las aulas: docentes y alumnos no están preparados para la educación a distancia.

${ }^{3}$ La página web https://www.aprendeencasa.mx se utilizó para crear presentaciones e infografías que servían como actividades para los alumnos de siguientes niveles: inicial (que incluye a lactantes e infantes de maternal), Preescolar, Primaria, Secundaria, Educación especial y Educación básica para adultos, que debían ser adjuntadas a un portafolio de evidencias que debían ser revisadas por los docentes al regreso a clases, cuando se tenía previsto el regreso el $1^{\circ}$ de junio de 2020 (AEF, 2020). 
EEI sitio web fue un intento apresurado de la SEP para emular un $\mathrm{MOOC}^{4}{ }^{4}$ que durante el periodo inicial previsto de dos semanas lectivas de aislamiento solo estaba conformado por actividades extraídas directamente de los libros de texto gratuitos ${ }^{5}$ y adaptadas a una presentación visualmente poco atractiva en la que no existía una interacción alumno-docente, como tampoco elementos interactivos o de autogestión que lo hicieran interesante para los estudiantes; en algunos casos se agregaban recomendaciones de videos de YouTube, lecturas adicionales y referencias al libro de texto de donde se tomaron los temas sugeridos (Navarrete, Manzanilla, Ocaña, 2020). Conforme se vio la necesidad de ampliar la cuarentena, la página web se fue modificando: se aumentó el material de estudio y se agregaron enlaces con otras páginas existentes de organizaciones no gubernamentales como PruebaT ${ }^{6}$ o Chango Maniaco, ${ }^{7}$ inter alia.

Sin demeritar el esfuerzo hecho por las autoridades educativas, quienes pusieron un empeño considerable en la rápida implementación del sitio web Aprende en Casa para dar continuidad a la enseñanza, es innegable que aún hace falta trabajar arduamente en el proyecto para responda a las necesidades de los usuarios y, sobre todo, con su objetivo primordial: servir como paliativo ante la ausencia de la educación tradicional. Pero la realidad es dolorosamente visible: sin una intervención concreta, el rezago aumentará, principalmente en poblaciones pobres y vulnerables..., considerando que el uso de las tecnologías sigue siendo "muy débil" respecto al impacto que puede tener si se considera que puede usarse para estas actividades, no solo las recreativas, y que los contenidos no están actualizados; eso sin contar que los hogares aún tienen deficiencias de conectividad (Cárdenas, 2020, en Ortega, 2020a).

Una intervención necesaria es replantear el paradigma educativo actual, reformular la necesidad de una educación a distancia como modalidad permanente y la actualización de la formación de docentes con asignaturas que incluyan la enseñanza a distancia, puesto que precisamente la formación y capacitación docente es parte de los factores que han imposibilitado el proceso de enseñanza en esta modalidad.

${ }^{4}$ MOOC son las siglas en inglés de Massive Online Open Courses (Cursos Online Masivos y Abiertos). Es decir, se trata de un curso a distancia, accesible por Internet, al que se puede apuntar cualquier persona y no tiene límite de participantes. Además de los materiales de un curso tradicional, como son los videos, lecturas y cuestionarios, los MOOC proporcionan forums de usuarios interactivos que ayudan a construir una comunidad para los estudiantes, profesores y los teaching assistants (https://www.uab.cat/web/estudiar/mooc/-que-es-un-cursomooc-1345668281247.html).

${ }^{5}$ La Comisión Nacional de Libros de Texto Gratuitos (CONALITEG) es el organismo público descentralizado de la Administración Pública Federal que para cada ciclo escolar produce y distribuye de manera gratuita los libros de texto que requieren los estudiantes inscritos en el Sistema Educativo Nacional (CONALITEG, 2020).

${ }_{6}^{6}$ PruebaT es una plataforma en línea desarrollada por la Fundación Carlos Slim, cuyo objetivo es brindar de manera gratuita experiencias de aprendizaje a estudiantes, docentes y padres de familia, con el fin de fortalecer conocimientos y habilidades para aprender a lo largo de la vida. https://pruebat.org/

${ }^{7}$ Chanco Maniaco es una plataforma de software educativo, que enlaza con plataformas de aprendizaje en línea como: twig eduation, little bridge y mangahigh. https://changomaniaco.com/

Diálo@os sobre Educación 


\section{La actitud docente ante la educación a distancia}

La premura con la que se tomaron las medidas para el cierre de escuelas, así como una coordinación deficiente entre las autoridades educativas federales y estatales, tuvieron como resultado esfuerzos paupérrimos para permitir el seguimiento del currículo que pudiera servir como apoyo a alumnos y padres durante las semanas de confinamiento (Fernández et al., 2020). Maestros y maestras viven agobiados por un sistema económico, político, social y cultural, nacional y global, que desconoce su realidad; desde antes de la pandemia, la escuela tradicional era incapaz de cubrir las necesidades de los alumnos. La vinculación entre escuela y maestros se encuentra debilitada y fracturada, existen demasiadas restricciones institucionales y sociales para desarrollar la labor docente (Romero, 2020). Los docentes, una vez más, enfrentan las decisiones de la autoridad educativa con más iniciativa que técnica, con sus propios recursos y encarando las limitaciones de un sistema que poco puede ofrecer una capacitación docente adecuada para incorporar las TIC en la experiencia pedagógica cotidiana, programas con un costo de miles de millones de pesos para la adquisición de computadoras, laptops, tabletas y distintas tecnologías de las que hoy pocos pueden echar mano para acceder a la educación en línea, y la insuficiente adaptación de los materiales didácticos de las diferentes asignaturas para su enseñanza en línea (Fernández, 2020).

Los docentes promedio de educación básica no tienen una formación que incluya asignaturas que los prepare para la enseñanza a distancia (SEP, 2012), las medidas tomadas por las autoridades educativas para dar continuidad al ciclo escolar no consideraron las carencias y limitaciones formativas que los docentes enfrentan en su labor cotidiana, lo que los coloca en una situación de desventaja para cubrir las horas clase con contenido de calidad. Ya iniciado el periodo de aislamiento, la SEP y la empresa Google se unieron para brindar capacitación virtual a docentes y padres de familia en una plataforma auxiliar para la educación a distancia, con la meta inicial de 500 mil maestros y padres de familia (SEP, 2020), con el objetivo de llegar a un millón de maestros y padres en todos los niveles de educación (Ortega, 2020), pero tan solo para el cuarto trimestre de 2019 se registraron 1'197,778 docentes en educación básica, de los cuales, más de la mitad se ocupa del nivel de educación primaria (INEGI, 2020).

Sobre el porcentaje de docentes a nivel nacional que implementaron la educación a distancia, la Ciudad de México quedó muy por debajo del Estado de México o de la zona norte del país (gráfica 1), ya sea porque muchos de los docentes no se sentían preparados (gráfica 2) o por la falta de apoyo por parte de los directivos o autoridades para realizar su función educativa (gráfica 3) (Valora, 2020).

Diálo@os 


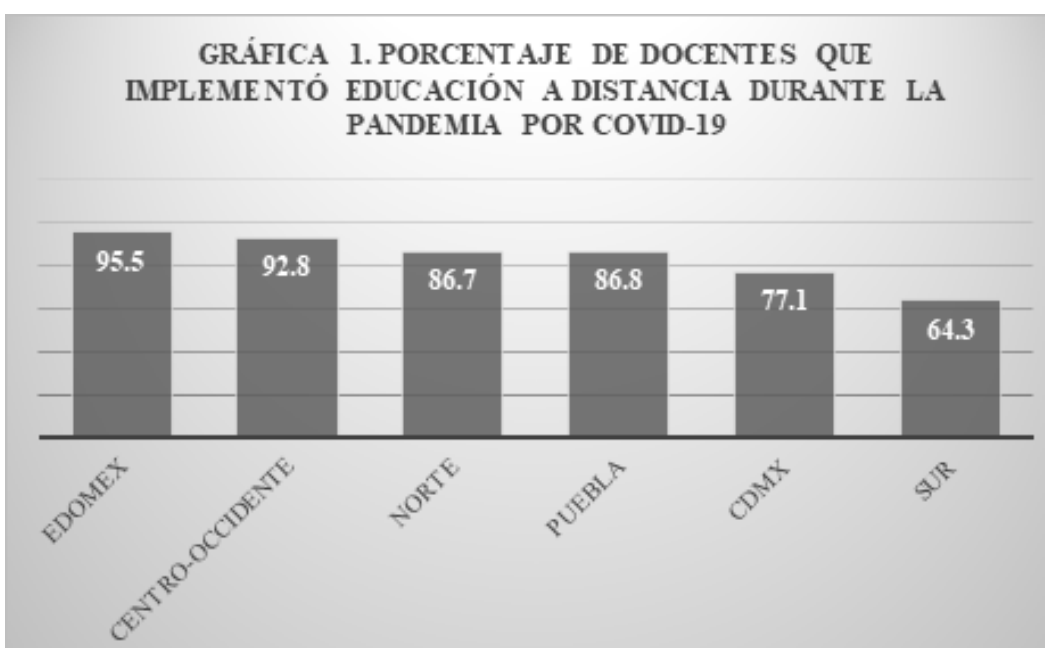

Fuente: Valora, 2020: 5.

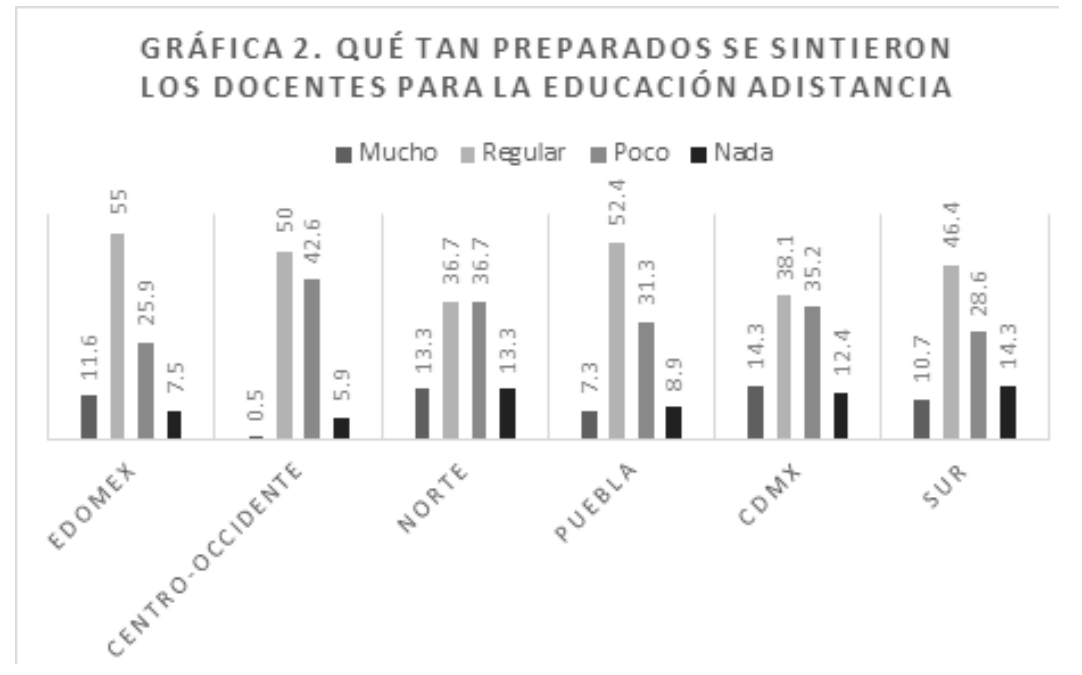

Fuente: Valora, 2020: 6. 


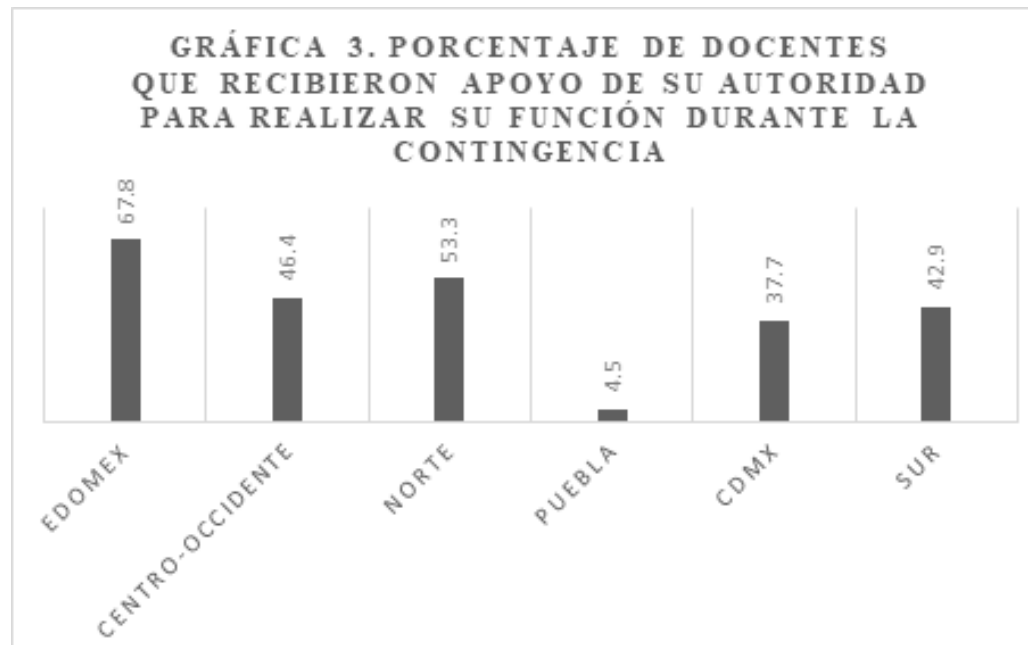

Fuente: Valora, 2020:7.

Es evidente que existen múltiples factores por los que los docentes no pudieran dar clases a distancia, pero una de las más representativas es la falta de capacidades y habilidades digitales necesarias para la enseñanza a distancia. Lo cual pone de manifiesto la necesidad de una capacitación docente para educar a distancia que comience con su formación inicial y que continúe a lo largo de toda la vida, pues normalmente los programas gubernamentales que han tratado de integrar las TIC en la educación implementan primero el programa y posteriormente se da la capacitación a los docentes; por otro lado, casi todos los programas establecidos por el gobierno federal desde $1997^{8}$ hasta el último en 2016 , se enfocan en los alumnos de $5^{\circ}$ y $6^{\circ}$ grado de primaria, ${ }^{9}$ olvidando las necesidades de los alumnos de $1^{\circ}$ a $4^{\circ}$ (SEP, 2016: 29-39). El periodo actual de aislamiento social dejó al descubierto una dolorosa verdad: la educación básica en México no está preparada para la educación a distancia. A pesar de los recursos, tanto financieros como humanos, empleados en los programas de promoción de las TIC, poco se ha avanzado hacia la inclusión digital.

\section{Los alumnos: de la reclusión en las aulas al confinamiento en los hogares}

Pese a que ya existía una crisis global de aprendizajes, pues aun cuando los estudiantes se encontraban en las escuelas era evidente que no estaban adquiriendo las habilidades fundamen-

${ }^{8}$ Son varios los programas que ha impulsado el gobierno federal desde 1997, iniciando con el programa Red Escolar, que estuvo vigente de 1997 a 2004, seguido por Enciclomedia, vigente de 2004 a 2011. Posteriormente, se da inicio al programa Habilidades Digitales para Todos, que estuvo vigente de 2009 a 2012, el programa Mi Compu.mx cuya vigencia fue de 2013 a 2014, seguido por el Programa Piloto de Inclusión Digital de 2013 a 2015 y, finalmente, el programa @prende cuya duración fue de 2014 a 2016 (SEP, 2016: 29-39).

${ }^{9}$ Casi todos los programas de integración a las TIC en educación primaria se han enfocado en dar cobertura a $5^{\circ}$ y $6^{\circ}$ de primaria, con excepción del programa Red Escolar (1997-2004) y Habilidades Digitales para Todos (2009-2012), cuyo alcance era para Primaria y Secundaria (SEP, 2016: 39).

Diálo@os 
tales necesarias para la vida: $53 \%$ de los niños de 10 años en países de ingresos bajos y medianos no pueden leer ni comprender un texto simple (Saavedra, 2020). La actual crisis sanitaria hará más profundas las carencias educativas y el rezago, sobre todo en la Educación Básica. Los alumnos de pronto tienen que enfrentar una modalidad educativa a la que no están acostumbrados, para la cual no han desarrollado las habilidades digitales necesarias y en la que muchos no tienen acceso; por ello, más que una educación a distancia se consideraría una educación mediada por TIC.

Bajo la premisa utópica de salvar el ciclo escolar (Martínez, 2020), el secretario de Educación Pública se olvida de que las aulas se han convertido en espacios de confinamiento donde los alumnos asisten en horarios cada vez más extensos, usan los libros de texto, toman notas y presentan exámenes, pero no desarrollan aprendizajes significativos. El cambio de las aulas a los hogares ha puesto en evidencia que la labor educativa ha menguado hasta hacerse casi inexistente (Ortega, 2020), por lo que el enfoque de una educación a distancia bajo la supervisión paternal queda solo en buenas intenciones.

Para los padres que siguieron sus actividades en el campo laboral formal, el cuidado de los hijos y el seguimiento académico se complicó considerablemente, volviéndose una empresa imposible cuando se carece de redes de apoyo para el cuidado de los menores; aquellos que trabajan en casa enfrentan dificultad en la distribución del tiempo entre las demandas laborales, familiares y escolares, mientras que aquellos que sobreviven en el empleo informal se vieron mayormente perjudicados por la pérdida repentina de sus ingresos y, aún más, cuando no pudieron llevar a cabo su labor por tener que hacerse cargo de sus hijos e hijas (De la Cruz, 2020).

La falta de una política establecida por la SEP, encargada de robustecer la relación escuela-hogar, ha propiciado una falta de conciencia sobre la complementariedad que hay entre los esfuerzos que realizan los docentes en la escuela y la importancia de las actividades en el hogar (Santizo, 2020). Mientras que los padres que cuentan con un nivel educativo medio superior o superior tienen mayor participación en el acompañamiento de las actividades escolares de sus hijos e hijas, este va disminuyendo en relación con el nivel educativo de los padres, es decir, entre menor sea el nivel alcanzado por los padres menor es el acompañamiento que realizan, al mismo tiempo que se observa una disminución de dicho acompañamiento conforme aumenta el nivel educativo de los hijos (Sánchez et al., 2010).

Así pues, los alumnos deben enfrentar no solo las carencias de acceso a la tecnología y materiales necesarios para seguir estudiando, sino también carencias de apoyo por parte de sus maestros y tutores. Si bien en México se está proporcionando servicios educativos a través de la estrategia Aprende en Casa con el sitio web del mismo nombre, canales de televisión nacional, la radio y canales digitales, con programas basados en el plan nacional de estudios (UNICEF, 2020), estos han resultado insuficientes para cubrir las necesidades de una población estudiantil que ha vivido en crisis desde hace décadas. 
Es importante reconocer que la crisis por COVID-19 nos proporciona una gran oportunidad de ampliar la educación a distancia a la educación básica, por medio de un modelo planteado desde un diseño instruccional basado en una pedagogía que proporcione las habilidades digitales para todos, que incluya no solo a los niños, niñas y jóvenes de primaria y secundaria, sino que permita disminuir la brecha digital de los docentes y padres de familia y creando una red de enseñanza mucho más equitativa e incluyente.

\section{Implicaciones de un sistema organizado de Educación Básica a Distancia}

La educación en México, desde principios del siglo XX ha sido materia de debate y los temas parecen no cambiar: la educación indígena, la diferencia entre educación e instrucción, la necesidad de una educación integral y con valores (Granja, 2010), a lo que hoy se suma la necesidad de un nuevo paradigma educativo. Ante la necesidad de preparar al sector educativo para un regreso a la nueva normalidad, la emergencia sanitaria por el COVID-19 puso en evidencia la poca preparación que se tiene para enfrentar una crisis de cualquier tipo; sin embargo, las bases de un proyecto de enseñanza a distancia para la educación básica ya fueron puestas en marcha, a pesar de la premura con la que se realizó; no obstante, es evidente la necesidad de un replanteamiento que sirva como base para un nuevo sistema educativo, más dinámico, efectivo y global, y que impulse el desarrollo integral de los alumnos a cualquier nivel educativo. Las implicaciones para un programa de este tipo son varias, pero considerablemente positivas en sus aspectos generales de implementación y mantenimiento.

La educación a distancia implica la flexibilización de la acción educativa en el tiempo y el espacio que, con el uso de las TIC, se ha convertido en un sistema virtual de educación que desde mediados del siglo XX y hasta principios del XXI se enfocó a los estudios universitarios (Olea, 2002; Navarrete, Manzanilla, 2017). Pero no debe enfocarse en el uso de tecnologías futuras, sino invertir esfuerzos en construir modelos de enseñanza para obtener mejores resultados con las tecnologías que se tienen actualmente, pues la innovación no se alcanza mediante la novedad sino mediante la aplicación de criterios para conseguir nuevos escenarios formativos y comunicativos (Ramírez, 2016).

Incremento de la cobertura. De acuerdo con el informe del Instituto Nacional para la Evaluación de la Educación (INEE) (2019), pese a la cobertura casi universal en educación básica, aún existe un porcentaje considerable de niños, niñas y jóvenes que no asisten a la escuela: en las áreas rurales la inasistencia es mayor (17.1\%) que en las áreas urbanas (13.5\%), lo que se traduce en 1.5 millones de niños y niñas en áreas rurales y 3.3 millones en áreas urbanas que no asisten a la escuela. Por otro lado, casi 2.5 millones de niñas y niños en pobreza no asisten a la escuela, poco más de medio millón de niños indígenas no tienen acceso a la escuela y aproximadamente 208,000 niños con discapacidad enfrentan mayores retos para acceder a la educación obligatoria (INEE, 2019: 36-43).

Diólo pos

sobre Educación

temas aCtUALES EN INVESTIGACION EDUCA año 12 | número 22 | enero-junio 2021 | ISSN 2007-2171 
La ED puede ampliar la cobertura de la Educación Básica a estos subsectores que aún se encuentran fuera de la escuela, ya sea por limitaciones en la movilidad o por limitaciones de acceso a las escuelas físicas. La implementación de la educación a distancia no requiere de una inversión tan grande como la que se necesitaría para un espacio físico (INEE, 2007), la ED requiere de medios o recursos técnicos que permitan la comunicación salvando tiempo y distancia, reduciendo obstáculos geográficos, económicos, de trabajo y familiares que pudieran afectar a los estudiantes (Alfonso, 2003).

Aumento en la calidad. Establecida la brecha de disponibilidad y oportunidad para los servicios educativos que atienden a la población indígena, migrante, de alta marginación o con discapacidad, destaca la falta o la insuficiencia de personal capacitado para atenderlos, a pesar de que en todos los tipos de escuela hay estudiantes que presentan algún tipo de discapacidad o tienen alguna necesidad educativa especial; en primaria, alrededor de $67 \%$ de las escuelas tiene por lo menos un estudiante con discapacidad, y solo en 33.8\% de ellas hay personal que proporcione algún tipo de atención (INEE, 2019).

Las características intrínsecas de la ED permiten la incorporación de herramientas de comunicación que posibiliten a especialistas académicos la implementación de recursos enfocados a estos subsectores de población en edad escolar de acuerdo a las necesidades de cada segmento, que permitan a los alumnos conocer y utilizar infinidad de programas, recursos y herramientas que ayuden y faciliten la comprensión y el aprendizaje, que desarrollen, entre otras, la habilidad de comprensión lectora (indispensable para cualquier sistema educativo) (Salazar, 2019); también es sabido que la ED favorece el grado de alfabetización digital al proporcionar información actualizada y pertinente a las necesidades educativas actuales (Pacheco, s/f), aumentando así la calidad en la educación al permitir enfoques calificados a problemas específicos.

Diversidad de esquemas pedagógicos. Los sistemas de ED proporcionan oportunidades para establecer modelos educativos en los que el aprendizaje adquiera un sentido diferente respecto del que se tiene en los sistemas presenciales. Mediante adecuaciones curriculares que se ajusten a los perfiles de los alumnos, crear modelos educativos centrados en el estudiante que, en la realidad, no se han extendido tanto como deberían (Moreno, Cárdenas, 2012).

La ED permite respetar los estilos y modos de aprendizaje de los alumnos, tomando en cuenta sus condiciones de vida y estudio, fomentando la libertad del alumno en un ambiente y entorno propicios para elegir contenidos de acuerdo a sus necesidades e intereses. Cada institución que ha incursionado en la modalidad a distancia lo ha hecho considerando las características particulares de su filosofía educativa, su práctica y sus experiencias, lo cual nos hace notar que no existe un esquema único, ni siquiera un estilo definido, no obstante sus ventajas y desventajas (Ávalos, 2002), pero abre la posibilidad de aprovechar las áreas de oportunidad para una mejora continua. 
Fortalecimiento de la investigación. La investigación educativa es, sin lugar a dudas, fundamento esencial del quehacer educativo; por eso, ante los cambios acelerados del conocimiento y la pluralidad de paradigmas, se necesita de profesionales competentes que den respuesta a la problemática actual, capaces de adoptar una posición profesional al investigar una realidad compleja y dinámica, y trasformarla de manera creativa (Puebla, 2014).

Las oportunidades de investigación dentro del campo de la ED son muchas, dado que es una modalidad relativamente nueva, más aún en la Educación Básica a Distancia, donde no se ha puesto tanto énfasis en el uso de las herramientas virtuales y aún se siguen los paradigmas educativos de finales del siglo pasado. El propósito principal de cualquier plan de estudios es el de generar información para incidir en la mejora de las prácticas de enseñanza de los docentes y el aprendizaje de los estudiantes; optimizar la planeación y la toma de decisiones de las autoridades escolares para la mejora de las condiciones y la efectividad del currículo, y el de elaborar directrices para la toma de decisiones en política pública educativa (INEE, 2019).

La Educación Básica a Distancia como un recurso de valor comprobado. La EBD tiene como objetivo el alcance de los segmentos de la población que por motivos culturales, sociales o económicos no se adapta o no tiene acceso a los sistemas convencionales de educación (Mendoza, Campos, Rivera, 2020). No se pretende sustituir la función formadora de las instituciones educativas, sino ser parte de una evolución de la misma para conseguir una modalidad que dé mayor cobertura y eleve la calidad de la educación básica en México.

La Educación Básica a Distancia debe comprometerse a satisfacer las necesidades de gestión, generación y aplicación de conocimiento que demanda la delineación y restructuración de las prácticas organizacionales (Avendaño, 2012:60), para proporcionar a su vez herramientas que ayuden a la educación tradicional para implementar el uso cotidiano de las tecnologías en la educación.

Evolución de los roles de los actores educativos. Los roles tradicionales de la educación habrán de evolucionar en la EBD; por un lado, el alumno deberá desarrollar capacidades de planeación y organización para delimitar el tiempo que dedicará a cada asignatura, siendo un agente activo en su proceso de formación, controlando y evaluando los contenidos, ambientes y actividades de aprendizaje de forma que la experiencia sea dinámica y contribuya a la edificación de conocimiento (Moreno, Cárdenas, 2012). El docente, por su parte, debe ser capaz de manejar la información digital evaluando su finalidad y relevancia, comunicarse en entornos digitales con seguridad, proteger sus datos e identidad y, a su vez, la integridad de sus pupilos; debe crear contenidos nuevos, integrar y relaborar conocimientos y contenidos previos; y ser capaz de solucionar problemas, identificar necesidades y recursos digitales acorde a la finalidad o necesidad (Viñals, Cuenca, 2016).

Dadas las implicaciones enumeradas, consideramos que vivimos el momento oportuno para implementar un sistema de EBD. Los beneficios de esta modalidad educativa superan las

Diólo@os sobre Educación TEMAS ACTUALES EN INVESTIGACION : año 12 | número 22 | enero-junio 2021 | ISSN 2007-2171 
limitaciones que se pudieran presentar en su implementación, los problemas organizativos o estructurales que pudieran presentarse pueden salvarse satisfactoriamente una vez que diera inicio el proyecto, lo cual es una de las ventajas del Diseño Instruccional de Gagné y Briggs, tal como lo veremos en el siguiente apartado.

\section{Propuesta de implementación de un programa permanente de Educación a Distancia}

Muchas voces se han levantado en favor y en contra de esta nueva realidad educativa, pero los avances siempre han despertado susceptibilidades en aquellos que se encuentran en su zona de confort, los grandes cambios siempre han traído consigo detractores que desean mantener todo dentro de lo ya conocido, de lo ya probado; aunque cierto es que los grandes cambios no se suceden de la noche a la mañana, también es cierto que la pasividad nunca ha traído nada nuevo, nada histórico, nada reformador. La EBD no significa el vaticinio del fin de la escuela tradicional ni de la desaparición de la docencia; implica la evolución de la institución educativa conoci$\mathrm{da}$, significa encontrar un punto de convergencia que permita la coexistencia de la modalidad presencial y la modalidad a distancia en una interacción continua para trasformar la Educación Básica en México.

\section{Lo primero: el diseño instruccional para una nueva realidad educativa}

La base fundamental para cualquier modalidad educativa es el diseño instruccional. Cuando un profesional se plantea el desarrollo de un curso sigue un proceso, de forma consciente o rutinaria, con el fin de diseñar y desarrollar acciones formativas de calidad. El disponer de modelos que guíen este proceso es de indudable valor para el docente o el pedagogo, que será requerido para diseñar los materiales y estrategias didácticas del curso (Belloch, s/f).

Existen numerosas definiciones de diseño instruccional. En los siguientes párrafos presentaremos algunas de ellas, por ejemplo, para Reigeluth, una teoría de diseño instruccional ofrece una guía explícita de cómo ayudar a las personas a desarrollar mejores aprendizajes. Los tipos de aprendizaje pueden incluir desarrollo cognitivo, emocional, social, físico y espiritual (Reigeluth, 2009). Una aproximación sistemática para desarrollar aprendizaje mediado y presencial, que incluya una declaración del curso y los objetivos de rendimiento del aprendizaje terminal, evaluación de los objetivos y retroalimentación a los estudiantes y al docente para determinar en qué medida se dominaron los objetivos y qué tan bien funcionaron las estrategias seleccionadas de enseñanza/aprendizaje (Simonson, Seepersaud, 2019).

El diseño instruccional en la web utiliza la atención y motivación a través de ligas a una base de conocimiento existente (Cassarino, 2014). El diseño instruccional es el arte y la ciencia aplicada de crear un ambiente instruccional y los materiales, claros y efectivos, que ayudarán al alumno a desarrollar la capacidad para lograr ciertas tareas (Broderick, 2001, citado en Pa- 
checo, s/f). Podemos definir el diseño instruccional como el proceso sistemático, continuo y pedagógico en el cual se fundamentan las distintas teorías instruccionales y psicopedagógicas de aprendizaje, con el fin de cumplir los objetivos planteados, mediante el análisis específico de necesidades. Para promover y conducir el desarrollo y creación de materiales de enseñanza, como actividades educativas, interactivas e instruccionales, así como también de pruebas y evaluaciones (Pacheco, $\mathrm{s} / \mathrm{f}$ ).

Para efectos del presente trabajo, nos enfocaremos en el modelo instruccional eclécti$\mathrm{Co}^{10}$ de Gagné y Briggs (Gagné, Briggs, Wagner, 1974), mismo que se sustenta en las teorías de aprendizaje estímulo-respuesta y de modelos de procesamiento de información (Belloch, s/f), mediante la asociación entre los elementos cognitivo-conductuales (Cárdenas, 2015); con una secuencia instruccional fija, prestablecida y basada en el procesamiento de la información (Feo, Guerra, 2013).

\section{El modelo instruccional de Gagné y Briggs para la Educación Básica a Distancia}

El modelo de Gagné y Briggs (figura 1) se divide en cuatro niveles: 1. Nivel de sistema, 2. Nivel de curso, 3. Nivel de lección y 4. Nivel de sistema final (ITSON, s/f), que integran los 14 pasos que contiene y que describiremos a continuación:

${ }^{10}$ Cabe señalar que no hay disciplinas ni teorías puras, con una esencia e identidad última, sino que están contaminadas por desarrollos procedentes de otros campos. Se entiende por eclecticismo aquello que podemos elegir de entre varias teorías (disciplinas, perspectivas, metodologías, etc.), y que ese elegir no es buscar o sacar "lo mejor de cada teoría", sino solo aquello que nos sea útil y compatible ontológica y epistémicamente entre lo que pretendamos elaborar en pro de las mejores herramientas teóricas, analíticas y metodológicas para el análisis de la "realidad social", por tanto, ser ecléctico no implica per se un sentido sincrético (cfr. Navarrete, 2016).

\section{Diólo os}


Figura 1. Modelo instruccional de Gagné y Briggs (1974)

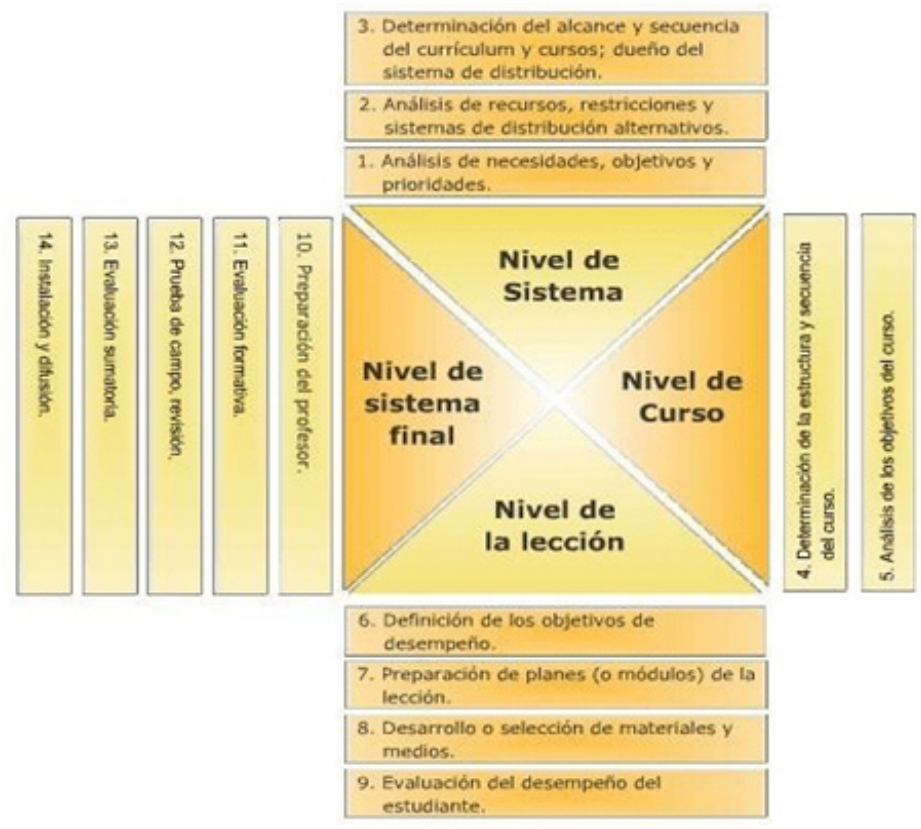

Fuente: ITSON, s/f.

Nivel de sistema. El nivel de sistema comienza con el paso 1. Análisis de necesidades, objetivos y prioridades, con el fin de establecerlas y determinar sus implicaciones instruccionales. Paso 2. El análisis de recursos, restricciones y sistemas de distribución alternativos: establece los métodos, materiales y actividades que se consideran factibles para alanzar los objetivos. Paso 3. En la determinación del alcance y secuencia del currículum y cursos: dueño del sistema de distribución, se diseña el alcance y el planteamiento de la secuencia didáctica, estableciendo los objetivos en términos de desempeño y determinando los detalles del sistema de distribución que se va a desarrollar.

El primer paso deberá ser analizado y establecido a través de la acción pedagógica, tomando como base el plan de estudios vigente para la educación básica, pero considerando la flexibilidad necesaria para la inclusión de los sectores analizados previamente. Es importante que durante el análisis del plan de estudios se elimine en lo posible el material repetitivo e innecesario, para dar lugar a material relevante como el cuidado del medio ambiente, la educación digital, etc.; mientras que los pasos 2 y 3 quedan a cargo de los diseñadores que darán forma a las necesidades, objetivos y prioridades plasmadas dentro del análisis inicial, y cuyo esfuerzo se trasformará en los materiales y actividades necesarios para que los alumnos alcancen las metas establecidas, pero tomando en consideración sus necesidades específicas de aprendizaje, lenguaje o limitaciones; para estos puntos, los diseñadores deberán crear actividades que puedan 
ser dobladas a lenguas indígenas, lenguaje de señas mexicano, etc., que sea dinámico y atractivo, lo que atraería la atención de los alumnos, así como considerar el uso del gaming ${ }^{11}$ para los temas del plan de estudios que así lo permitan.

Nivel de Curso. Paso 4. Determinación de la estructura y secuencia del curso: se refiere a la organización de los materiales instruccionales y las actividades en un orden lógico de desarroIlo, con el fin de facilitar el aprendizaje sistémico, en primer lugar, por unidades y desglosando cada una por objetivos. En esta etapa se propone el desarrollo de actividades que incluyan la opción multilenguaje con las 11 familias lingüísticas mexicanas, ${ }^{12}$ así como adecuaciones para alumnos con discapacidad, por ejemplo, deberá incluir archivos de audio para alumnos con discapacidad visual o actividades dinámicas e interactivas para alumnos con déficit de atención. Paso 5. Análisis de los objetivos del curso: mediante el cual se realizará la categorización de los resultados del aprendizaje e identificación de las condiciones de aprendizaje que tendrán que ser establecidas. Aquí es importante considerar las condiciones específicas de cada sector de población identificado, en relación con los objetivos de aprendizaje que se buscan, por ejemplo, para los alumnos con discapacidad visual no pueden dirigirse actividades visuales en pantalla, como memoramas, crucigramas, rompecabezas o similares; deberán estar enfocadas en formatos de audio, tipo podcast, que puedan seguir en libros braille.

Nivel de la lección. Paso 6. Definición de los objetivos de desempeño: se establecen los objetivos específicos de cada lección, mismos que guiarán el desarrollo instruccional y los mecanismos de evaluación necesarios para valorar la efectividad; por ejemplo, deben considerar los objetivos específicos para alumnos con discapacidad cognitiva, que no pueden ser iguales a los de un alumno sin discapacidad; los objetivos serán determinados a nivel pedagógico y desarrollados por los diseñadores. Paso 7. Preparación de los planes (o módulos) de la lección: los módulos deberán incluir una instrucción basada en un grupo, conducida por el docente, en el que cada objetivo de la lección haga una lista de los eventos instruccionales, identifique los materiales, medios o agentes necesarios para aplicarlos, especifique los planes para todas las actividades de aprendizaje, incluyendo la forma en que se utilizarán los medios y materiales, y la forma en que el docente intervendrá en cada lección. Paso 8. Desarrollo o selección de materiales y medios: implica el diseño o elección de los materiales que ayuden a los participantes a cumplir los objetivos. Paso 9. Evaluación del desempeño del estudiante: implica la preparación de medidas de desempeño para determinar si los alumnos alcanzan los objetivos. Las rúbricas

${ }^{11}$ El gaming es la ejecución de aplicaciones especializadas conocidas como juegos electrónicos o videojuegos en consolas de videojuegos o computadoras personales (como el conocido como juego en línea). (McGonigal, 2015).

${ }^{12}$ Una familia lingüística es un conjunto de lenguas cuyas semejanzas obedecen a un origen común; una agrupación lingüística es el conjunto de variantes comprendidas bajo el nombre dado tradicionalmente a un pueblo indígena; variante es una forma de hablar que presenta diferencias en su estructura, comparada con la variante de la misma agrupación. En el país existen 11 familias lingüísticas, divididas en 68 agrupaciones de la que derivan 364 variantes lingüísticas https://www.gob.mx/inali, por lo anterior, y debido a la complejidad de dar mantenimiento a cada variante dentro de una plataforma, se propone sentar solo las familias lingüísticas.

Diólopos 
de evaluación deberán considerar el nivel de los alumnos, así como los objetivos de aprendizaje y estar, en caso necesario, en la lengua materna del alumno.

Nivel de sistema final. Este nivel del modelo de Gagné y Briggs, que incluye los pasos 10 al 14, aplica solo a los diseñadores de sistemas de aprendizaje globales, pues consiste en el diseño de métodos para entrenar a los profesores en el uso del nuevo sistema, llevar a cabo la evaluación formativa por medio de cursos pilotos de evaluación o lecciones individuales en unas cuantas clases para identificar las necesidades de revisión. Paso 10. Preparación del profesor: considerando que se trataría de un sistema desconocido para los docentes, se establece la necesidad de un curso preparatorio que incluya los conocimientos básicos para el manejo de una plataforma MOODLE, así como el diseño de herramientas y actividades de enseñanza. Paso 11. Evaluación formativa: deberá incluir cursos subsecuentes y clases de nivelación para mantener actualizado al docente. Paso 12. Prueba de campo, revisión: para lo cual es necesario que los docentes realicen lecciones individuales que permitan revisar si los objetivos específicos, materiales y evaluaciones cumplen los objetivos generales previstos del diseño instruccional. Paso 13. Evaluación sumatoria: se refiere a la aplicación del diseño instruccional en lo que se relaciona a cada paso del diseño, si este cumple o no con las proyecciones hechas en las etapas de planeación y desarrollo. Finalmente, una vez evaluados los pasos anteriores y corregidos los fallos que pudieran haberse detectado, se procede al paso 14. Instalación y difusión del programa de Educación Básica a Distancia.

\section{Conclusiones}

En respuesta a las preguntas que dieron origen a esta investigación: ¿es posible estructurar un proyecto permanente de educación a distancia para la educación básica? ¿la modalidad a distancia permitiría una mayor inclusión y cobertura en la educación básica? y ¿es viable establecer la modalidad a distancia a partir de la educación básica primaria y secundaria?, consideramos que: dadas las condiciones actuales que se viven a nivel mundial, nos encontramos ante la posibilidad de reformar integralmente la Educación Básica en México, es el momento oportuno para establecer de manera permanente una modalidad a distancia para la educación básica, que no solo permita la cobertura educativa en tiempos de crisis sino que pueda establecerse como una opción permanente que logre dar cobertura a los grupos de población que por razones económicas, físicas o culturales no asisten a la escuela.

Estimamos que el diseño instruccional de Gangé y Briggs, propuesto para el modelo de EBD, cubre los puntos necesarios para dar inicio al programa, pues permite la evaluación y modificación de los puntos que lo estructuran conforme se va avanzando en el diseño. Por otro lado, la implementación de un programa a distancia no requiere de una inversión tan importante como se necesita para la construcción y administración de espacios físicos. El ciclo escolar 2020-2021 inició en línea, pero al momento no incluye capacitación para los maestros en ma- 
teria de ED (SEP, 2020a), mientras tanto, se seguirá con la instrucción mediada por TIC, una vez más, sin subsanar las carencias de una población estudiantil que, año tras año, va acumulando deficiencias en su formación.

Históricamente, el docente se ha visto en la necesidad de cubrir las carencias del profesiograma, ${ }^{13}$ sin capacitación, sin recursos, sin apoyo institucional; por lo que se espera que en esta ocasión asuma una vez más un rol para el que "no está preparado". Ser docente no es una labor sencilla, se requiere de un nivel de compromiso, ética y valores que muchas veces van más allá de los muros de la institución educativa. Su identidad se ha ido desdibujando en las últimas décadas, han enfrentado no solo reformas educativas que acrecentaron su estrés laboral, sino también la persecución laboral, como fue el caso de la Reforma Educativa de Enrique Peña Nieto, en la que se les responsabilizó de las carencias en la educación del país.

La presente propuesta se enfoca a la Educación Básica como un programa completo que permita dar continuidad a la educación en tiempos de crisis, como la pandemia por COVID-19, en los que la educación presencial no es viable. Sin embargo, también se considera que esta propuesta puede funcionar como un programa permanente, que permitiría la integración de todos los sectores de la población estudiantil que por razones de discapacidad, pobreza extrema o problemática social (como niños migrantes, indígenas no hispanohablantes o niños que trabajan), no tienen acceso a la educación básica presencial, dando acceso a una educación en la que la inclusión y la cobertura darían un enorme paso hacia la universalización de la educación básica.

En esta propuesta, la educación a distancia también daría voz y presencia a alumnos de educación especial, que contarían con material especialmente diseñado de acuerdo a sus capacidades y necesidades específicas, sin tener que trasladarse a un espacio físico, eliminando así las diferencias sociales para aquellos que no cuentan con los recursos necesarios para movilizarse hacia una institución educativa. Por otro lado, la educación rural, indígena y migrante podría verse beneficiada con la EBD, mediante el diseño de cursos en su lengua materna relacionados con su cultura y estilo de vida, que permitan su integración de manera natural, evitando la deserción por frustración.

Si bien estamos conscientes de las limitaciones tecnológicas existentes en muchas regiones del país, como comunidades sin servicio de energía eléctrica, sin Internet y, más aún, familias que no tienen siquiera un teléfono móvil con acceso a Internet (cfr. Navarrete, Manzanilla, Ocaña, 2020); no obstante, a pesar de estas deficiencias, consideramos que los beneficios superarían los costes de implementación de la EBD. En su inicio la implementación se realizaría

${ }^{13} \mathrm{El}$ profesiograma es un documento técnico-administrativo que organiza la interrelación, interactuación e interdependencia de un puesto de trabajo desde tres puntos de vista: el de Gestión del Talento Humano, el de Seguridad Ocupacional y el de Salud Laboral, en el cual se resumen las aptitudes y capacidades de los puestos de trabajo que existen y los que cumplen los trabajadores (López, s/f: 3).

Diálo@os sobre Educación año 12 | número 22 | enero-junio 2021 | ISSN 2007-2171 
en las áreas donde las condiciones así lo permitieran, con el fin de medir y evaluar el alcance y pertinencia de un programa como el que se propone.

Se considera que el Diseño Instruccional propuesto por Gagné y Briggs permitiría la implementación de un modelo de EBD capaz de cubrir las necesidades educativas de los alumnos del nivel de educación básica. Mediante la revisión de cada paso en el proyecto, es posible la corrección o adecuación del programa, y de esta manera, alcanzar un incremento en la cobertura y la calidad de la educación básica en México.

\section{Referencias}

Adhanom-Ghebreyesus, T. (2020). Alocución de apertura del Director General de la OMS en la rueda de prensa sobre la COVID-19 celebrada el 11 de marzo de 2020. https://www.who.int/ es/dg/speeches/detail/who-director-general-s-opening-remarks-at-the-media-briefingon-covid-19---11-march-2020

Administración Educativa Federal (AEF). (2020). Actividades para reforzar los aprendizajes esperados durante el aislamiento preventivo. Aprende en Casa. https://www.aprendeencasa. $\underline{\mathrm{mx}}$

Alfonso-Sánchez, I. R. (2003). La educación a distancia. ACIMED, 11. La Habana, Cuba. http://scielo. sld.cu/scielo.php?script=sci arttext\&pid=S1024-94352003000100002

Ávalos López, E. (2002). Implicaciones pedagógicas de la educación a distancia. https://ibp6.webnode.es/ files/200000082-0d22e0e206/IMPLICACIONES\%20PEDAGOGICAS\%20DE\%20 LA\%20EDUCACION\%20A\%20DISTANCIA-ERNESTO\%20AVALOS.pdf

Avendaño-Porras, V. del C. (2012). Alcances y posibilidades de la educación a distancia en la sociedad del conocimiento. El caso de la Universidad Virtual del Estado de Guanajuato. Revista Mexicana de Bachillerato a Distancia, 4(7). http://www.revistas.unam.mx/index.php/ $\mathrm{rmbd} /$ article/view/44486/40187

Belloch, C. (s/f). Diseño Instruccional. Unidad de Tecnología Educativa. España: Universidad de Valencia. https://www.uv.es/bellochc/pedagogia/EVA4.pdf

Biblioteca ITSON (s/f). Modelos de diseño instruccional. http://biblioteca.itson.mx/oa/educacion/ oa32/moldelos diseno instruccional/z3.htm

Cárdenas-Porras, K. N. (2015). Modelo de Gagné y Briggs. https://prezi.com/nuhvayd9faq3/modelo-de-gagne-y-briggs/

Cassarino, C. (2014). Instructional Desing Priciples for an e-learning Enviroment: A Call for Definitions in the Field. Querterly Review of Distance Education; Charlotte, 4(4), 455-461. https://search.proquest.com/openview/81a68ddc796781119d059706c5a5eee1/1?pqorigsite $=$ gscholar $\&$ cbl $=29705$ 
CONALITEG (2020). Misión de la Comisión Nacional de Libros de Texto Gratuitos. https://www.gob. $\mathrm{mx} /$ conaliteg/que-hacemos

De la Cruz-Flores, G. (2020). El hogar y la escuela: lógicas en tensión ante la COVID-19. Educación, escuela y continuidad pedagógica. Educación y pandemia. Una visión académica. México: IISUE-UNAM. https://www.iisue.unam.mx/investigacion/textos/educacion pandemia.pdf

Dirección General de Planeación y Programación (DGPP) (2008). Glosario. Términos utilizados en la Dirección General de Planeación y Programación. México: DGPP/SEP. http://cumplimientopef.sep.gob.mx/2010/Glosario\%202008\%2024-jun-08.pdf

Feo, R.; C. Guerra (2013). Propuesta de un modelo de diseño instruccional para la elaboración e implementación de cursos a distancia en el Instituto Pedagógico de Miranda José Manuel Siso Martínez. Sapiens, Revista Universitaria de Investigación, 14(1), 65.83. https://www.redalyc.org/pdf/410/41059088002.pdf

Fernández, M. A.; L. Herrera; D. Hernández; R. Nolasco; R. De la Rosa (2020). Lecciones del COVID-19 para el sistema educativo mexicano. Nexos Distancia por tiempos. Blog en educación. https://educacion.nexos.com.mx/?p=2228

Gagné, R. M.; L. Briggs; W. W. Wagner. (1974). Principles of Instructional Design. 4th ed. Harcourt Brace College Publishers. Library of Congress Cataloging-in-Publication Data. https://www. hcs64.com/files/Principles\%20of\%20instructional\%20design.pdf

Galeana-Cisneros, R. (2016). Identificación de subpoblaciones de niños que no asisten a la escuela y caracterización de los factores asociados a la problemática. Conformando un marco inicial para la construcción de indicadores. Estadísticas e indicadores temáticos. México: INEE. https://www.inee.edu.mx/wp-content/uploads/2019/01/P3B105.pdf

Granja-Castro, J. (2010). Procesos de escolarización en los inicios del siglo XX. La instrucción rudimentaria en México. Perfiles Educativos, 32(129). http://www.scielo.org.mx/scielo. php?script=sci arttext\&pid=S0185-26982010000300005

Instituto Nacional para la Evaluación de la Educación (INEE) (2007). Infraestructura escolar en las primarias y secundarias de México.https://www.inee.edu.mx/wp-content/uploads/2019/01/ P1D231.pdf

Instituto Nacional para la Evaluación de la Educación (INEE) (2019). La educación obligatoria en México 2019. https://www.inee.edu.mx/wp-content/uploads/2019/04/P1|245.pdf

Instituto Nacional de Estadística y Geografía (INEGI) (2020). Estadísticas a propósito del Día del Maestro (Docentes en Educación Básica en México. Datos nacionales). Comunicado de prensa Número 215/20, 13 de mayo de 2020. https://www.inegi.org.mx/contenidos/saladeprensa/aproposito/2020/EAP Maestro2020.pdf

Instituto Nacional de la Lengua Indígena (2020). Estadística básica. http://site.inali.gob.mx/Micrositios/estadistica basica/ 
López E., I. (s/f). Profesiogramas, herramienta proactiva para prevenir riesgos laborales. Uniminuto. Corporación Universitaria Minuto de Dios. Educación de calidad al alcance de todos, Seccional Bello. http://www.uniminuto.edu/documents/991974/2604818/IVAN+LOPEZ+-+Los tprofesiogramas, ,herramienta+proactiva+para+prevenir+riesgos+laborale.pdf/

Martínez, L. (2020). Vamos a salvar el ciclo escolar de manera presencial: Esteban Moctezuma. SDPnoticias-nacional. 01 de mayo de 2020. https://www.sdpnoticias.com/nacional/esteban-moctezuma-regreso-a-clases-cuarentena-coronavirus-covid-19.html

McGonigal, J. (2015). Los juegos online pueden crear un mundo mejor. Video de youtube, series TED. https://youtu.be/dE1DuBesGYM

Mendoza-Becerril, M. A.; H. Campos-Cruz; C. Rivera-Pérez (2020). Educación a distancia en México: Centros Públicos de Investigación. ID NotiCyTI. https://noticyti.com/opinion/963educaci\%C3\%B3n-a-distancia-en-m\%C3\%A9xico-centros-p\%C3\%BAblicos-deinvestigaci\%C3\%B3n.html

Moctezuma-Barragán, E. (2020) Acuerdo número 02/03/20 por el que se suspenden las clases en las escuelas de educación preescolar, primaria, secundaria, normal y demás para la formación de maestros de educación básica del sistema educativo nacional, así como aquellas de los tipos medio superior y superior dependientes de la Secretaría de Educación Pública. Diario Oficial de la Federación. https://www.dof.gob.mx/nota detalle.php?codigo=558947 9\&fecha $=16 / 03 / 2020$

Moctezuma-Barragán, E. (2020b) Acuerdo número 06/03/20 por el que se amplía el periodo suspensivo del 27 de marzo al 30 de abril del año en curso y se modifica el diverso número 02/03/20 por el que se suspenden las clases en las escuelas de educación preescolar, primaria, secundaria, normal y demás para la formación de maestros de educación básica del Sistema Educativo Nacional, así como aquellas de los tipos medio superior y superior dependientes de la Secretaría de Educación Pública. Diario Oficial de la Federación. https:// dof.gob.mx/nota detalle.php?codigo $=5590981 \&$ fecha $=01 / 04 / 2020$

Moctezuma-Barragán, E. (2020c) Acuerdo número 09/04/20 por el que se amplía el periodo suspensivo del 23 de marzo al 30 de mayo del año en curso y se modifica el diverso número 02/03/20 por el que se suspenden las clases en las escuelas de educación preescolar, primaria, secundaria, normal y demás para la formación de maestros de educación básica del Sistema Educativo Nacional, así como aquellas de los tipos medio superior y superior dependientes de la Secretaría de Educación Pública. Diario Oficial de la Federación. https:// dof.gob.mx/nota detalle.php?codigo $=5592554 \&$ fecha $=30 / 04 / 2020$

Moctezuma-Barragán, E. (2020d). Acuerdo número 12/06/20 por el que se establecen diversas disposiciones para evaluar el ciclo escolar 2019-2020 y cumplir con los planes y programas de estudio de educación básica (preescolar, primaria y secundaria), Normal y demás para la formación de maestros de educación básica aplicables a toda la república, al igual que 
aquellos planes y programas de estudio del tipo medio superior que la Secretaría de Educación Pública haya emitido, en beneficio de los educandos. Diario Oficial de la Federación. https://dof.gob.mx/nota detalle.php?codigo $=5594561 \&$ fecha $=05 / 06 / 2020$

Moctezuma-Barragán, E.; M. Bucio-Mújica (2020). Boletín N 80. Fortalece SEP programa Aprende en Casa mediante sitio web especializado en educación básica. Gobierno de México-Secretaría de Educación Pública. Blog. https://www.gob.mx/sep/articulos/boletin-no-80-fortalece-sep-programa-aprende-en-casa-mediante-sitio-web-especializado-en-educacionbasica?idiom $=$ es

Moreno-Almazán, O.; M. G. Cárdenas-López (2012). Educación a distancia: nueva modalidad, nuevos alumnos. Perfiles de alumnos de Psicología en México. Perfiles Educativos, 34(136). http://www.scielo.org.mx/scielo.php?script=sci arttext\&pid=S0185-26982012000200008

Nación (05 de febrero 2020). Cierran 974 planteles de "La Escuela es Nuestra" por falta de alumnos. La Silla Rota. https://lasillarota.com/nacion/cierran-974-planteles-de-la-escuela-esnuestra-por-falta-de-alumnos $/ 358970$

Navarrete, Z. (2016) Re-discusión del eclecticismo teórico en las ciencias sociales, humanas y de la educación. En Ducoing, P. (coord.). La investigación en educación. Epistemologías y metodologías. México: Plaza y Valdés/Asociación Francófona Internacional de Investigación Científica en Educación, 329-339.

Navarrete, Z;; H. Manzanilla (2017). Panorama de la educación a distancia en México. Revista Latinoamericana de Estudios Educativos, 13(1), 65-82. http://190.15.17.25/latinoamericana/ downloads/Latinoamericana13(1) 4.pdf

Navarrete, Z.; H. Manzanilla; L. Ocaña (2020). Políticas implementadas por el gobierno mexicano frente al COVID-19. El caso de la educación básica. Revista Latinoamericana de Estudios Educativos, 50 (Número especial), 143-172. https://rlee.ibero.mx/index.php/rlee/article/ view/100/474

Olea-Deserti, E. (2002). La educación a distancia ¿Modalidad educativa moderna? Investigación Administrativa, 31(90). https://www.ipn.mx/assets/files/investigacion-administrativa/docs/ revistas/90/ART4.pdf

Organización Mundial de la Salud (OMS) (2009). Alerta y respuestas mundiales. Gripe por A (H1N1): preguntas frecuentes. https://www.who.int/csr/disease/swineflu/faq/es/\#whatis

Ortega, A. (2020). Aprender 'on line' en medio de la pandemia incrementa rezago educativo. Expansión Política. https://politica.expansion.mx/mexico/2020/04/27/aprender-on-lineen-medio-de-la-pandemia-incrementa-rezago-educativo

Ortega, A. (2020a). Con dudas y poco apoyo, así enfrentan maestros la enseñanza en tiempos de COVID. Expansión Política. https://politica.expansion.mx/mexico/2020/05/15/con-dudas-ypoco-apoyo-asi-enfrentan-maestros-la-ensenanza-en-tiempos-de-covid 
Pacheco, M. (s/f). Diseño instruccional: desarrollo y adaptación de contenidos para la formación a distancia "Curso comunicación familiar". Tésis de licenciatura. Universidad Pedagógica Nacional. http://200.23.113.51/pdf/28997.pdf

Puebla-Espinosa, A. (2014). Importancia de la investigación educativa. Ponencia presentada en el Primer Congreso Internacional de Transformación Educativa. https://www.transformacioneducativa.com/index.php/articulos-sobre-educacion/54-importancia-de-la-investigacioneducativa

Ramírez-León, R. H. (2016). Los retos que impone la educación a distancia en México. Revista lberoamericana de Producción Académica y Gestión Educativa, 3(6). https://www.pag.org.mx/ index.php/PAG/article/view/631

Reigeluth, Ch. M. (2009). Instructional-Desing Theories and Models. A new Paradigm of Instructional Theory, Vol. II. Nueva York: Library of Congress. https://books.google.com.mx/bo oks?hl=en\&lr=\&id=OWavJCNfhcsC\&oi=fnd\&pg=PP1\&dq=instructional+design+\%22 theories+and+models\%22\&ots=28TyNMaX2d\&sig=PGDEqrsFaCO32amzJ8hi9mh7Ug $4 \&$ redir esc=y\#v=onepage\&q=instructional\%20design $\% 20 \% 22$ theories $\% 20$ and $\% 20$ models $\% 22 \& \mathrm{f}=$ false

Saavedra, J. (2020). COVID-19 y educación: Algunos desafíos y oportunidades. Education for Global Development. Banco Mundial. https://blogs.worldbank.org/es/education/educationalchallenges-and-opportunities-covid-19-pandemic

Salazar-Muñoz, Z. X. (2019). Análisis del modelo de educación a distancia en México. https:// www.gestiopolis.com/analisis-del-modelo-de-educacion-a-distancia-en-mexico/

Salgado Andrade, E.; F. Villavicencio Zarza (2009). Crónica de una epidemia pregonada. http:// www.scielo.org.mx/scielo.php?script=sci arttext\&pid=S1607-050X2010000100007

Sánchez-Escobedo, P.; Á. Valdés-Cuervo; M. Reyes-Mendoza; E. Carlos-Martínez (2010). Participación de padres de estudiantes de educación primaria en la educación de sus hijos en México. Liberabit, 16(1). http://pepsic.bvsalud.org/scielo.php?script=sci arttext\&pid $=$ S1729-48272010000100008

Santizo-Rodall, C. (2020). Foro virtual de análisis. La educación básica y los desafíos impuestos por el COVID-19 y el confinamiento sanitario. Consejo Mexicano de Investigación Educativa. http://www.comie.org.mx/v5/sitio/2020/05/14/foro-virtual-de-analisis-la-educacionbasica-y-los-desafios-impuestos-por-el-covid-19-y-el-confinamiento-sanitario/

SEP (2012). Plan de estudio 2012. Malla curricular. Licenciatura en educación primaria plan 2012. https://www.dgespe.sep.gob.mx/reforma curricular/planes/lepri/malla curricular

SEP (2016). @prende 2.0. Programa de inclusión digital 2016-2017. Capítulo III Antecedentes. https://www.gob.mx/cms/uploads/attachment/file/162354/NUEVO PROGRAMA PRENDE 2.0.pdf 
SEP (2020). Inicia SEP, en colaboración con Google, capacitación virtual de más de 500 mil maestros y padres de familia. Boletín No 101. Gobierno de México Blog. https://www.gob.mx/sep/ articulos/boletin-no-101-inicia-sep-en-colaboracion-con-google-capacitacion-virtual-demas-de-500-mil-maestros-y-padres-de-familia?idiom=es

SEP (2020a). Consejo Técnico Escolar 2020-2021, Guía de trabajo. Fase intensiva. Educación preescolar, primaria y secundaria. México: Subsecretaría de Educación Básica. https://anexosprofelandia.files.wordpress.com/2020/07/ctefi-2021.pdf

Simonson, M.; D. Seepersaud (2019). Distance Education. Definition and Glossary of Terms. 4th ed. Charlotte: Information Age Publishing. https://books.google.com.mx/books?hl=e $\underline{n} \& \mid r=\& i d=K V V u D w A A Q B A J \& o i=f n d \& p g=P R 7 \& d q=i n s t r u c t i o n a l+d e s i g n+S i m o n s o n \%$ C2\%B4s+definition\&ots=FgaYq712Yq\&sig=OkrhDsyR31nccceof7us1SWDBuM\&redir esc $=\mathrm{y} \# \mathrm{v}=$ onepage $\& \mathrm{q}=$ instructional\%20desing $\& \mathrm{f}=$ false

UNICEF (2020). La falta de igualdad en el acceso a la educación a distancia en el contexto de la COVID-19 podría agravar la crisis mundial del aprendizaje. Comunicado de prensa 05 de junio de 2020. https://www.unicef.org/es/comunicados-prensa/falta-igualdad-acceso-educaciondistancia-podria-agravar-crisis-aprendizaje

Valora (2020). Docencia en tiempos de pandemia. Sondeo de prácticas docentes y participación familiar en educación básica, durante la suspensión de clases en el periodo del 20 de marzo al 20 de abril de 2020 (Fase 1 de la pandemia). Valora Consultoría SC. https://valora.com.mx/ wp-content/uploads/2020/05/200424-sondeo-educaci\%C3\%B3n-en-pandemia.pdf

Viñals-Blanco, A.; J. Cuenca-Amigo (2016). El rol docente en la era digital. Revista Interuniversitaria de Formación del Profesorado, 30(2). https://www.redalyc.org/jatsRepo/274/27447325008/ html/index.html 